\title{
PRAKTISCHE ASPEKTE VON RASSENVERGLEICHSVERSUCHEN BEIM RIND
}

\section{F. WeBer. - Institut für Tierzucht, E.T.H. Universität str. 2, Zürich (Schweiz).}

Am Beispiel von Ergebnissen schweizerischer Rassenvergleichsversuche werden folgende vier Fragen diskutiert :

I. Welche Rassen sollen miteinander verglichen werden?

2. Worauf soll sich der Vergleich beziehen?

3. Wo und wie soll der Vergleich durchgeführt werden?

4. Wie sollen die Ergebnisse in der züchterischen Praxis verwertet werden?

Die Einordnung der Rassen nach bestimmten Kriterien in eine Reihe erleichtert die Beurteilung der Versuchsergebnisse. Vergleiche unselektionierter, miteinander nicht verwandter Tiere auf Station erlauben es, die Rassenunterschiede in einzelnen Eigenschaften, nicht aber im Gesamtwert einwandfrei zu ermitteln. Der Vergleich zwischen reinrassigen Tieren und Kreuzungstieren wird durch die Heterosis verfälscht. Die Heterosis und die maternalen Effekte sind aus dem Vergleich verschiedener Kreuzungsstufen im Feld zu schätzen. Die Genotyp-Umweltinteraktion Rasse $\times$ Land lässt sich durch Versuche in einem Land allein nicht bestimmen; die Interaktion Stier $\times$ Land ist meist bedeutungslos. An Stelle oder neben eigentlichen Rassenvergleichsversuchen wird der routinemässige Einbau von Stieren fremder Rassen in die eigenen Nachzuchtprüfungsprogramme empfohlen, da wir oft an Stiereneffekten ebenso interessiert sind wie an Rasseeffekten.

\section{METHODISCHE ASPEKTE UND ERGEBNISSE}

DER VERGLEICHE EINIGER MILCHRIND-UND

\section{ZWEINUTZUNGSHERKÜNFTE SOWIE DEREN KREUZUNGEN}

G. Schönmuth, G. Seeland, A. Wilke. - Boveich Tierziuchtung und Haustiergenetik HumboltUniversität, Berlin (DDR).

Der Verwendung fremder Rassen in Zuchtprogrammen sollten Prüfungen vorausgehen, die eindeutige Aussagen über die zu erwartenden Vor-und Nachteile solcher Rassen zuiassen. Diese Prüfungen erfordern einen hohen Aufwand und demzufolge eine fundierte Planung.

Zur Prüfung von Milchrassen bieten sich prinzipiell zwei Methoden an :

I) der Import weiblicher Tiere, mit folgender Vergleichsprüfung

2) der Import von Sperma oder Bullen und Vergleich der Kreuzungsgenerationen unter sich bzw. mit einer Lokalrasse.

Zwischen beiden Varianten bestehen dann ergänzende Verbindungen, wenn die weiblichen Tiere als tragende Färsen importiert und dadurch sogleich auch Bullen dieser Herkunft als Kälber im Mutterleib eingeführt werden.

Die Vergleichsprüfung, als Grundlage für die daraus resultierende zuchtmethodische Strategie, enthält viele Probleme und stellt sich als schwieriger heraus, als die Realisierung eines bestimmten Zieles selbst. kann :

Der Vergleich sollte methodisch so angelegt sein, dass er u. a. zwei Erfordernisse erfüllen

I) Klare Erkennung der Leistungsdifferenzen zwischen den Vergleichspartnern

2) Frühzeitige Erkennung dieser Differenzen.

Aus der Verbindung dieser beiden Erfordernisse, plädieren wir für eine umfassende Vergleichsprüfung importierter weiblicher Tiere unter prognostischen Produktionsbedingungen.

In der DDR wurden in zurückliegenden Jahren folgende Herkünfte im Vergleich zum Deutschen Schwarzbunten Rind (DSR) geprüft :

I. Schwarzbunte aus der BRD; vorwiegend aus Ostfriesland

2. Schwedische Schwarzbunte

3. Niederlandische Schwarzbunte (FRS und NRS)

4. Dänische Schwarzbunte

5. Britisch-Friesian $(B F)$

6. Kanadisch-Friesian $(H F)$ 
Zwischen den Herkünften I-4 konnten keine gesicherten Differenzen nachgewiesen werden, ebenso keine Úberlegenheit zur Vergleichspopulation.

Eindeutige Leistungsdifferenzen ergaben sich zwischen $B F$ und $H F$ einerseits zum $D S R$ andererseits, vor allem beim Vergleich weiblicher Originaltiere unter Versuchsbedingungen. Die Variation der Haltung (Laufstall-Anbindehaltung) veränderte diese Aussage nicht. Einer sehr hohen Leistungsüberlegenheit in den Milchmengen, der Euterform und Melkbarkeit steht bei den HF ein Minus im Fett-und Eiweissgehalt gegenuber. Hieraus ergaben sich Konsequenzen für das Zuchtprogramm der DDR.

In der Mastleistung erwiesen sich die Friesian-Herkünfte als mindestens gleichwertig zum DSR, bei geringer Unterlegenheit in der Schlachtleistung.

Diese eindeutigen Aussagen, besonders hinsichtlich der Differenzen zwischen $B F$ und $H F$, sind anhand der Zuchtwertschätzung unseres Materials allein nicht möglich.

\section{GENETISCHE DIFFERENZEN ZWISCHEN FRIESIANS}

IN U. S. A. UND BUNDESREPUBLIK DEUTSCHLAND

\section{O. GRAVERT. - Institut für Milcherzeugung, Kiel (BRD).}

Zur Bestimmung der genetischen Differenzen zwischen Holstein Friesians in U. S. A. und Schwarzbunten in der Bundesrepublik Deutschland wurden vier HF-Bullen mit bekanntem Zuchtwert eingesetzt und 46 Töchter mit 34 vergleichbaren Tieren deutscher Abstammung verglichen. Nach Korrektur für väterliche Abstammung ergab sich ein Schätzwert für die genetischen Differenzen zwischen den Populationsdurchschnitten von $674 \mathrm{~kg}$ Milch, 0,06 Prozent Fett und $24 \mathrm{~kg}$ Fett. Fũr die Mastleistung ergaben sich Schätzwerte von +30 g tägliche Zunahme, -2 Prozent Ausschlachtung und - 70 DM Schlachtwert.

\section{STRAIN DIFFERENCES IN MIIKING PERFORMANCE, FOOD CONSUMPTION AND BODY SIZE AMONGST FRIESIAN CATTLE IN DANISH TEST STATIONS}

\section{A. Zarnecki ('), C. J. M. Hinks*. - Institute of Animal Genetics, Edinburgh. * ARC Animal Breeding Research Organisation, Edinburgh.}

The purpose of this study was to examine and attempt to quantify differences in milking performance between progeny of Danish, Swedish, German, NRS and FRS sires located in Danish progeny test stations. 244 sire groups were examined. Least squares analyses of variance of daughter group means revealed strain differences in milk yield, the largest of which occurred between the Danish SDM and the German strain. Since no significant differences in protein p. Ioo and fat p. Ioo were apparent, observed differences in fat and protein yields could be ascribed only to differences in milk yield. There were no significant differences in body weight amongst the strains examined.

It was suggested that the observed differences in milking performance may have arisen from different selection practices in different countries of origin but that further experimental studies are required to resolve the question. (Poland).

(1) On leave from the Department of Genetics and Animal Breeding, Academy of Agriculture, Cracow

\section{COMPARAISON DES CRITÈRES DE PRODUCTIVITÉ NUMÉRIQUE ET PONDÉRALE DE TROIS RACES BOVINES}

\section{A VIANDE FRANÇAISES}

B. Bibe, J. N. Bonnet, G. Cavagne, F. Ménissier, J. Sapa. - Département de Génétique animale, C. N. R. Z., 78350, Jouy en Josas (France).

Depuis plusieurs années les races à viande françaises sont largement utilisées pour leur valeur paternelle en croisement terminal. Par contre, les comparaisons de leur valeur maternelle sont 